\title{
High Accuracy Combination Method for Solving the Systems of Nonlinear Volterra Integral and Integro-Differential Equations with Weakly Singular Kernels of the Second Kind
}

\author{
Lu Pan, ${ }^{1}$ Xiaoming $\mathrm{He}_{,}{ }^{2}$ and Tao Lï ${ }^{1}$ \\ ${ }^{1}$ College of Mathematics, Sichuan University, Chengdu, Sichuan 610064, China \\ ${ }^{2}$ Department of Scientific Computing, The Florida State University, Tallahassee, \\ FL 32310, USA
}

Correspondence should be addressed to Xiaoming He, xhe2@fsu.edu

Received 21 October 2009; Accepted 1 April 2010

Academic Editor: Gradimir V. Milovanović

Copyright (C) $2010 \mathrm{Lu}$ Pan et al. This is an open access article distributed under the Creative Commons Attribution License, which permits unrestricted use, distribution, and reproduction in any medium, provided the original work is properly cited.

\begin{abstract}
This paper presents a high accuracy combination algorithm for solving the systems of nonlinear Volterra integral and integro-differential equations with weakly singular kernels of the second kind. Two quadrature algorithms for solving the systems are discussed, which possess high accuracy order and the asymptotic expansion of the errors. By means of combination algorithm, we may obtain a numerical solution with higher accuracy order than the original two quadrature algorithms. Moreover an a posteriori error estimation for the algorithm is derived. Both of the theory and the numerical examples show that the algorithm is effective and saves storage capacity and computational cost.
\end{abstract}

\section{Introduction}

In this paper, we first consider the following system of differential equations:

$$
\begin{gathered}
x_{i}^{\prime}(t)=f_{i}(t, x(t), z(t)), \\
x_{i}(0)=x_{i 0}, \quad i=1, \ldots, m,
\end{gathered}
$$

where $x(t)=\left(x_{1}(t), \ldots, x_{m}(t)\right), z(t)=\left(z_{11}(t), \ldots, z_{1 m}(t), \ldots, z_{m 1}(t), \ldots, z_{m m}(t)\right)$, and $z_{i j}(t)=$ $\int_{0}^{t}(t-s)^{\alpha_{i j}}(\ln (t-s))^{\beta_{i j}} k_{i j}(t, s, x(s)) d s, 0>\alpha_{i j}>-1, \beta_{i j}=0$ or $1, i, j=1, \ldots, m$ as well as $k_{i j}(t, s, x), i, j=1, \ldots, m$, are continuous functions for $0 \leq s \leq t \leq T$ and $x \in R^{m}$. 
The problem (1.1) can be transformed to the following system of nonlinear integral equations: find $z(t)$ and $x(t)$ satisfying

$$
\begin{aligned}
z_{i j}(t)= & \int_{0}^{t}(t-s)^{\alpha_{i j}}(\ln (t-s))^{\beta_{i j}} k_{i j}(t, s, x(s)) d s, \quad i, j=1, \ldots, m, \\
& x_{i}(t)=x_{i 0}+\int_{0}^{t} f_{i}(s, x(s), z(s)) d s, \quad i=1, \ldots, m,
\end{aligned}
$$

which is a special case of nonlinear Volterra integral system with weakly singular kernels of the second kind

$$
u_{i}(t)=y_{i}(t)+\sum_{j=1}^{m} \int_{0}^{t}(t-s)^{\alpha_{i j}}(\ln (t-s))^{\beta_{i j}} k_{i j}(t, s, u(s)) d s, \quad i=1, \ldots, m,
$$

where $-1<\alpha_{i j}<0$, and $k_{i j}(t, s, u)$ is continuous function on $0 \leq s \leq t \leq T$ and $u=\left(u_{1}, \ldots, u_{m}\right) \in R^{m}$.

Nonlinear Volterra integral and integro-differential equations with weakly singular kernels of the second kind play important roles in the mathematical modeling of many physical and biological phenomena, particularly in such fields as heat transfer, nuclear reactor dynamics, and thermoelasticity, in which it is necessary to take into account the effect of past history. Plenty of work has been done to develop and analyze numerical methods for solving the Volterra integral and integro-differential equations with weakly singular kernels of the second kind; see [1-5] and reference therein. The recent progress in this research area has been achieved for the extrapolation method [6], the spline collocation method [7], and the Galerkin method $[8,9]$. However, there are few works for solving the systems of these types of equations, which are more important than single equation for many applications. For example, the elastodynamic problems for piezoelectric and pyroelectric hollow cylinders under radial deformation can be successfully transformed into a system of two second kind Volterra integral equations.

The combination method as an accelerating convergence technique for solving integral equations was firstly presented in 1984 [10]. Similar to the extrapolation method, the combination method combines several approximations to obtain an approximation of higher accuracy. One important advantage of the extrapolation method and the combination method is parallel computation since those original approximations can be computed independently. However, the extrapolation method uses a coarse grid and some finer grids. We must do much more work on the finer grids than the coarse one, which lowers the degree of parallelism. On the other hand, the loads of computing the approximations in the combination method are close to each other. Hence the combination method is an efficient parallel method to obtain an approximation of high accuracy with a high degree of parallelism. Recently this method has been used to solve the first kind Abel integral equations [11]. In this paper, we will apply the high accuracy combination method for solving the systems of nonlinear Volterra integral and integro-differential equations with weakly singular kernels of the second kind, for which there are few results, even for the general convergence to be proved in Section 4 of this paper.

In this paper, based on Lyness's [12] modified mid-point rectangular quadrature rule and modified trapezoidal quadrature rule, we will construct two quadrature methods to solve 
the systems. By means of the asymptotic expansions of the errors for both algorithms, we present a high accuracy combination algorithm. Then by using the generalization of discrete Gronwall inequality [13], the convergence rate, stability, and the asymptotic expansion of the error of the combination approximate solution are proved. Comparing to other algorithms, for example, extrapolation methods [6, 14], the combination algorithm has the advantage of less computation complexity because both of the modified mid-point rectangular quadrature rule and the modified trapezoidal quadrature rule have the same step size. Moreover an a posteriori error estimation is obtained, by which we can rectify the accuracy of our algorithm in processing.

\section{Existence and Uniqueness of the Solution}

Since the uniqueness, existence, and numerical methods of (1.2) can be decided by (1.1), we only discuss the problem (1.3) in the following.

Let $k_{i j}(t, s, u)$ satisfy Lipschitz condition (A):

$$
\left|k_{i j}(t, s, u)-k_{i j}(t, s, v)\right| \leq L\|u-v\|_{\infty} \quad \forall u, v \in R^{m}, 0 \leq s \leq t \leq T, 1 \leq i, j \leq m,
$$

then there is a unique solution in (1.3). In fact $\alpha=\min _{i, j} \alpha_{i j}>-1, u(t)=\left(u_{1}(t), \ldots, u_{m}(t)\right)^{T}$, and $\|u(t)\|_{\infty}=\max _{1 \leq i \leq m}\left|u_{i}(t)\right|$. We can choose $\eta>0$ such that $\alpha-\eta>-1$. Thus we have

$$
N=\max _{1 \leq i, j \leq m, 0<t<T} t^{\alpha_{i j}-\alpha+\eta}\left|(\ln t)^{\beta_{i j}}\right|<\infty .
$$

Let $\left(C_{[0, T]}\right)^{m}=C_{[0, T]} \times \cdots \times C_{[0, T]}$ be a continuous function space which maps $[0, T]$ into $R^{m}$ and let $F$ be a mapping from $\left(C_{[0, T]}\right)^{m}$ to itself. Then we have

$$
F_{i}(u)(t)=y_{i}(t)+\int_{0}^{t} \sum_{j=1}^{m}(t-s)^{\alpha_{i j}}(\ln (t-s))_{i j}^{\beta} k_{i j}(t, s, u(s)) d s, \quad 1 \leq i \leq m,
$$

where $y=\left(y_{1}, \ldots, y_{m}\right) \in\left(C_{[0, T]}\right)^{m}$, and

$$
\begin{aligned}
& \left|F_{i}(u)(t)-F_{i}(v)(t)\right| \\
& \quad=\sum_{j=1}^{m} \int_{0}^{t}(t-s)^{\alpha-\eta}(t-s)^{\alpha_{i j}-\alpha+\eta}\left|(\ln (t-s))_{i j}^{\beta}\right|\left|k_{i j}(t, s, u(s))-k_{i j}(t, s, v(s))\right| d s \\
& \quad \leq \sum_{j=1}^{m} \int_{0}^{t}(t-s)^{\alpha-\eta} N L|u(s)-v(s)|_{\infty} d s \\
& \quad=m N L \int_{0}^{t}(t-s)^{\alpha-\eta}|u(s)-v(s)|_{\infty} d s, \quad 1 \leq i \leq m,
\end{aligned}
$$

where $f(t)=t^{\alpha-\eta} \in L_{[0, T]}^{\prime}$, which is due to $\alpha-\eta>-1$.

Lemma 2.1. Suppose that condition (A) is satisfied, then there is a unique solution to (1.3). 
Proof. Assume that $u(t)=\left(u_{1}(t), \ldots, u_{m}(t)\right)^{T}$ and $v(t)=\left(v_{1}(t), \ldots, v_{m}(t)\right)^{T}$ are two different solutions to (1.3). Defining $w(t)=u(t)-v(t)$, we get

$$
\begin{gathered}
w_{i}(t)=\sum_{j=1}^{m} \int_{0}^{t}(t-s)^{\alpha_{i j}}(\ln |t-s|)^{\beta_{i j}}\left(k_{i j}(t, s, u(s))-k_{i j}(t, s, v(s))\right) d s, \quad i=1, \ldots, m, \\
\|w(t)\|_{\infty} \leq m N L \int_{0}^{t} t^{\alpha-\eta}\|w(s)\|_{\infty} d s .
\end{gathered}
$$

Therefore using Gronwall inequality, we get $w(t)=0$, which leads to the uniqueness. $y(t)$ and

In order to prove existence, we use a simple iterative process: let $u^{(0)}(t)=0, u^{(1)}(t)=$

$$
u_{i}^{(n)}(t)=y_{i}(t)+\int_{0}^{t} \sum_{j=1}^{m}(t-s)^{\alpha_{i j}}(\ln (t-s))^{\beta_{i j}} k_{i j}(t, s) u^{(n-1)}(s) d s, \quad n=1,2, \ldots
$$

Now we will prove that $u^{(n)}(t)$ is a convergent sequence. Let

$$
V^{(n)}(t)=\left\|u^{(n)}(t)-u^{(n-1)}(t)\right\|_{\infty}
$$

then from (2.6) and (2.4) we get

$$
V^{(n+1)}(t) \leq M \int_{0}^{t}(t-s)^{\alpha-\eta} V^{(n)}(s) d s=M t^{\beta} * V^{(n)}=M^{n} t^{\beta} * \cdots * t^{\beta} * V^{(1)},
$$

where $t^{\beta} * V^{(n)}$ denotes convolution, $M=m N L$, and $\beta=\alpha-\eta$.

Taking Laplace transformation, we can easily deduce that

$$
\widehat{V}^{(n+1)}(t) \leq\left(M \frac{\Gamma(\beta+1)}{s^{\beta+1}}\right)^{n} \widehat{V}^{(1)}(s) .
$$

Taking inverse Laplace transformation, we get

$$
V^{(n+1)}(t) \leq \frac{(M \Gamma(\beta+1))^{n}}{\Gamma(n \beta+n)} \int_{0}^{t} \tau^{n(\beta+1)} V^{(0)}(t-\tau) d \tau
$$

Note that for $n>m$, we have

$$
u^{(n)}(t)-u^{(m)}(t)=\left(u^{(n)}(t)-u^{(n-1)}(t)\right)+\left(u^{(n-1)}(t)-u^{(n-2)}(t)\right)+\cdots+\left(u^{(m+1)}(t)-u^{(m)}(t)\right)
$$


or

$$
\left\|u^{(n)}(t)-u^{(m)}(t)\right\|_{\infty} \leq V^{(n)}(t)+\cdots+V^{(m+1)}(t)
$$

But using (2.10), we easily prove that

$$
\sum_{n=0}^{\infty} V^{(n+1)}(t) \leq \sum_{n=0}^{\infty} \frac{(M \Gamma(\beta+1))^{n}}{\Gamma(n \beta+n)} \int_{0}^{t} \tau^{n(\beta+1)} V^{(0)}(t-\tau) d \tau
$$

is a convergent series, which means that

$$
\lim _{n, m \rightarrow \infty}\left\|u^{(n)}(t)-u^{(m)}(t)\right\|_{\infty}=0 \quad \text { or } \quad u^{(n)}(t) \longrightarrow u(t)
$$

and $u(t)$ is the solution to (1.2).

\section{The Numerical Methods}

In this section two quadrature algorithms which are based on [12] will be given to solve the systems. Consider the weakly singular integral

$$
I(G)=\int_{a}^{b}(b-x)^{\alpha} g(x) d x, \quad G(x)=(b-x)^{\alpha} g(x)
$$

and Navot's modified trapezoidal rule [15]

$$
Q_{T}^{h}(G)=\frac{h}{2} G(a)+h \sum_{j=1}^{N-1} G\left(x_{j}\right)-\zeta(-\alpha) h^{1+\alpha} g(b) .
$$

There is an error estimate as follows:

$$
E_{T}^{h}(G)=Q_{T}^{h}(G)-I(G)=-\zeta(-\alpha-1) h^{2+\alpha} g^{\prime}(b)+O\left(h^{2}\right) .
$$

We differentiate with respect to $\alpha$ in (3.3) and get

$$
E_{T}^{h}\left(G^{\prime}\right)=Q_{T}^{h}\left(G^{\prime}\right)-I\left(G^{\prime}\right)=\left[\zeta^{\prime}(-\alpha-1)-\zeta(-\alpha-1) \ln h\right] h^{2+\alpha} g^{\prime}(b)+O\left(h^{2}\right) .
$$

Then we have

$$
I\left(G^{\prime}\right)=\int_{a}^{b}(b-x)^{\alpha}(\ln (b-x))^{\beta} g(x) d x, \quad G^{\prime}(x)=(\ln (b-x))^{\beta}(b-x)^{\alpha} g(x), \quad \beta=0,1
$$


such that

$$
Q_{T}^{h}\left(G^{\prime}\right)=\frac{h}{2} G^{\prime}(a)+h \sum_{j=1}^{N-1} G^{\prime}\left(x_{j}\right)-\left[-\beta \zeta^{\prime}(-\alpha)+\zeta(-\alpha)(\ln h)^{\beta}\right] h^{1+\alpha} g(b)
$$

Thus we have

$$
E_{T}^{h}\left(G^{\prime}\right)=Q_{T}^{h}\left(G^{\prime}\right)-I\left(G^{\prime}\right)=\left[\beta \zeta^{\prime}(-\alpha-1)-\zeta(-\alpha-1)(\ln h)^{\beta}\right] h^{2+\alpha} g^{\prime}(b)+O\left(h^{2}\right)
$$

Equations (3.5) and (3.6) are the integral functions with logarithm singularity and their modified trapezoidal rule, and (3.7) is an asymptotic expansion of the error.

From [16], we have the modified mid-point rectangular rule

$$
Q_{M}^{h}(G)=h \sum_{j=0}^{N-1} G\left(x_{j+1 / 2}\right)-\left(2^{-\alpha}-1\right) \zeta(-\alpha) h^{1+\alpha} g(b)
$$

Hence we get

$$
E_{M}^{h}(G)=Q_{M}^{h}(G)-I(G)=-\left(2^{-\alpha}-1\right) \zeta(-\alpha-1) h^{2+\alpha} g^{\prime}(b)+O\left(h^{2}\right)
$$

From (3.4)-(3.7), we get

$$
\begin{aligned}
E_{M}^{h}\left(G^{\prime}\right)= & Q_{M}^{h}\left(G^{\prime}\right)-I\left(G^{\prime}\right) \\
=- & {\left[-\beta 2^{-\alpha-1} \ln 2 \zeta(-\alpha-1)-\beta\left(2^{-\alpha-1}-1\right) \zeta^{\prime}(-\alpha-1)\right.} \\
& \left.\quad+\left(2^{-\alpha-1}-1\right) \zeta(-\alpha-1)(\ln h)^{\beta}\right] h^{2+\alpha} g^{\prime}(b)+O\left(h^{2}\right) .
\end{aligned}
$$

More generally we have

$$
\begin{aligned}
& Q_{M}^{h}\left(G^{\prime}\right) \\
& =h \sum_{j=0}^{N-1} G^{\prime}\left(x_{j+1 / 2}\right)-\left[-\beta 2^{-\alpha} \ln 2 \zeta(-\alpha)-\beta\left(2^{-\alpha}-1\right) \zeta^{\prime}(-\alpha)+\left(2^{-\alpha}-1\right) \zeta(-\alpha)(\ln h)^{\beta}\right] h^{1+\alpha} g(b) .
\end{aligned}
$$

Therefore from (3.7) and (3.10), if $\beta=0$, we get

$$
\left(2^{-\alpha-1}-1\right) E_{T}^{h}\left(G^{\prime}\right)-E_{M}^{h}\left(G^{\prime}\right)=O\left(h^{2}\right)
$$


or

$$
\left(\frac{1-2^{-\alpha-1}}{2-2^{-\alpha-1}} Q_{T}^{h}\left(G^{\prime}\right)+\frac{1}{2-2^{-\alpha-1}} Q_{M}^{h}\left(G^{\prime}\right)\right)-I\left(G^{\prime}\right)=O\left(h^{2}\right) .
$$

Note that the combination has a high accuracy order $O\left(h^{2}\right)$, which is higher than $O\left(h^{2+\alpha}\right)$ in (3.7) and (3.10).

If $\alpha=0, \beta=1$ in (3.5), then

$$
\begin{gathered}
Q_{T}^{h}\left(G^{\prime}\right)=\frac{h}{2} G^{\prime}(a)+h \sum_{j=1}^{N-1} G^{\prime}\left(x_{j}\right)+\frac{1}{2} \ln \left(\frac{h}{2 \pi}\right) h g(b), \\
Q_{M}^{h}\left(G^{\prime}\right)=h \sum_{j=0}^{N-1} G^{\prime}\left(x_{j+1 / 2}\right)-\frac{1}{2} \ln 2 h g(b),
\end{gathered}
$$

where $\zeta(0)=-0.5, \zeta^{\prime}(0)=-\ln (2 \pi) / 2$, and (3.13) becomes

$$
\left(\frac{1}{3} Q_{T}^{h}\left(G^{\prime}\right)+\frac{2}{3} Q_{M}^{h}\left(G^{\prime}\right)\right)-I\left(G^{\prime}\right)=O\left(h^{2}\right)
$$

Using (3.14) and (3.15), we can construct two algorithms for solving Volterra integral system of equations. Since the kernels of the systems are weakly singular, the following Navot's quadrature rule is used. Setting $t=t_{l}$, we get

$$
u_{i}\left(t_{l}\right)=y_{i}\left(t_{l}\right)+\sum_{j=1}^{m} \int_{0}^{t_{l}}\left(t_{l}-s\right)^{\alpha_{i j}}\left(\ln \left|t_{l}-s\right|\right)^{\beta_{i j}} k_{i j}\left(t_{l}, s, u(s)\right) d s, \quad i=1,2, \ldots, m .
$$

Now we recall the following lemma from [8].

Lemma 3.1. Let $g(t) \in C^{2 r}[a, b], G(x)=(b-t)^{\alpha}(\ln |b-t|)^{\beta} g(t), h=(b-a) / N, t_{k}=a+k h(k=$ $0, \ldots, N)$, then modified trapezoidal rule

$$
T_{N}(G)=\frac{h}{2} G\left(t_{0}\right)+h \sum_{j=1}^{N-1}-\left[-\beta \zeta^{\prime}(-\alpha)+\zeta(-\alpha)(\ln h)^{\beta}\right] g(b) h^{1+\alpha}
$$

has an asymptotic error expansion

$$
\begin{aligned}
E_{N}(G)= & \sum_{j=1}^{r-1} \frac{B_{2 j}}{(2 j) !} G^{(2 j-1)}(a) h^{2 j} \\
& +\sum_{j=1}^{2 r-1}(-1)^{j}\left[-\beta \zeta^{\prime}(-\alpha-j)+\zeta(-\alpha-j)(\ln h)^{\beta}\right] \frac{g^{(j)} h^{j+\alpha+1}}{j !}+O\left(h^{2 r}\right),
\end{aligned}
$$


where $\alpha>-1, \beta=0,1, \zeta(t)$ and $\zeta^{\prime}(t)$ are Riemann-Zeta function and its derivative function, and $B_{2 j}$ are Bernoulli numbers.

From the above, we obtain the following discrete system of equations for modified trapezoidal quadrature method:

$$
\begin{array}{r}
u_{i 0}=y_{i}\left(t_{0}\right), \\
u_{i l}=y_{i}\left(t_{l}\right)+\sum_{j=1}^{m}\left[h \sum_{k=0}^{l-1} w_{l k}\left(t_{l}-t_{k}\right)^{\alpha_{i j}} \times\left(\ln \left|t_{l}-t_{k}\right|\right)^{\beta_{i j}} k_{i j}\left(t_{l}, t_{k}, U_{k}\right)+w_{l l, i j} h^{1+\alpha_{i j}} k_{i j}\left(t_{l}, t_{l}, U_{l}\right)\right] \\
i=1,2, \ldots, m, \quad l=1,2, \ldots, N,
\end{array}
$$

where $h=1 / N, t_{k}=k h, U_{j}=\left(u_{1 j}, u_{2 j}, \ldots, u_{m j}\right), j=1,2, \ldots, m$, and

$$
w_{l k}= \begin{cases}\frac{1}{2} & \text { if } k=0, \\ 1 & \text { if } 1 \leq k \leq l-1, \\ \beta_{i j} \zeta^{\prime}\left(-\alpha_{i j}\right)+\zeta\left(-\alpha_{i j}\right)(\ln h)^{\beta_{i j}}-\beta_{i j} \zeta^{\prime}\left(-\alpha_{i j}\right) & \text { if } k=l, l=1,2, \ldots, N .\end{cases}
$$

On the other hand, we also obtain the discrete system for modified mid-point rectangular quadrature method

$$
\begin{aligned}
& u_{i 0}=y_{i}\left(t_{0}\right), \\
& u_{i l}=y_{i}\left(t_{l}\right)+\sum_{j=1}^{m}\left[h \sum_{k=0}^{l-1} w_{l k}\left(t_{l}-t_{k+1 / 2}\right)^{\alpha_{i j}}\right. \\
& \times\left(\frac{\left(\ln \left|t_{l}-t_{k+1 / 2}\right|\right)^{\beta_{i j}} k_{i j}\left(t_{l}, t_{k}, U_{k}+U_{k+1 / 2}\right)}{2}\right) \\
& \left.+w_{l l, i j} h^{1+\alpha_{i j}} k_{i j}\left(t_{l}, t_{l}, U_{l}\right)\right], \quad i=1,2, \ldots, m, l=1,2, \ldots, N,
\end{aligned}
$$

where

$$
w_{l k}= \begin{cases}1 & \text { if } 0 \leq k \leq l-1, \\ -\left[-\beta_{i j} 2^{-\alpha} \ln 2 \zeta(-\alpha)-\beta_{i j}\left(2^{-\alpha}-1\right) \zeta^{\prime}(-\alpha)\right. & \text { if } k=l, l=1,2, \ldots, N \\ \left.\quad+\left(2^{-\alpha}-1\right) \zeta(-\alpha)(\ln h)^{\beta_{i j}}\right] & \beta_{i j}=0, \text { or } 1 .\end{cases}
$$

Because the discrete system is nonlinear diagonal system of equations, we introduce the following iterative algorithms. 
Algorithm 3.2 (modified trapezoidal quadrature method). We have the following steps.

Step 1. Take $\varepsilon>0$ sufficiently small and let

$$
u^{0}\left(t_{j}\right)=\left(u_{1 j}^{0}, \ldots, u_{m j}^{0}\right)=\left(y_{1}\left(t_{j}\right), \ldots, y_{m}\left(t_{j}\right)\right), \quad j=1,2, \ldots, N
$$

and $n:=0$.

Step 2. Compute $u_{i l}^{n+1}(l \leq N, i=1,2, \ldots, m)$ in parallel by the following simple iteration:

$$
u_{i l}^{n+1}=y_{i}\left(t_{l}\right)+\sum_{j=1}^{m}\left[h \sum_{k=0}^{l-1} w_{l k}\left(t_{l}-t_{k}\right)^{\alpha_{i j}}\left(\ln \left|t_{l}-t_{k}\right|\right)^{\beta_{i j}} k_{i j}\left(t_{l}, t_{k}, U_{k}\right)+w_{l l} h^{1+\alpha_{i j}} k_{i j}\left(t_{l}, t_{l}, U_{l}^{n}\right)\right]
$$

where $U_{k}=\left(u_{1 k}, \ldots, u_{m k}\right), U_{l}^{n}=\left(u_{1 l^{n}}^{n}, \ldots, u_{m l}^{n}\right)$, and $w_{l k}$ is defined by (3.20).

Step 3. If $\max _{1 \leq i \leq m}\left|u_{i l}^{n+1}-u_{i l}^{n}\right| \leq \varepsilon$, then let $u_{i l}^{n+1}=\tilde{u}_{i l}$ and stop the iteration, otherwise set $n:=n+1$, go to Step 2 .

Algorithm 3.3 (modified mid-point rectangular quadrature method). We have the following steps.

Step 1. Take $\varepsilon>0$ sufficiently small and let

$$
\bar{u}^{0}\left(t_{j}\right)=\left(\bar{u}_{1 j}^{0}, \ldots, \bar{u}_{m j}^{0}\right)=\left(y_{1}\left(t_{j}\right), \ldots, y_{m}\left(t_{j}\right)\right), \quad j=1,2, \ldots, N
$$

and $n:=0$.

Step 2. Compute $\bar{u}_{i l}^{n+1}(l \leq N, i=1,2, \ldots, m)$ in parallel by the following simple iteration:

$$
\begin{array}{r}
\bar{u}_{i l}^{n+1}=y_{i}\left(t_{l}\right)+\sum_{j=1}^{m}\left[h \sum_{k=0}^{l-1} w_{l k}\left(t_{l}-t_{k}\right)^{\alpha_{i j}}\left(\ln \left|t_{l}-t_{k}\right|\right)^{\beta_{i j}}\right. \\
\left.\quad \times k_{i j}\left(t_{l}, t_{k}, \frac{\left(\bar{U}_{k}^{n}+\bar{U}_{k+1 / 2}^{n}\right)}{2}\right)+w_{l l, i j} h^{1+\alpha_{i j}} k_{i j}\left(t_{l}, t_{l}, \bar{U}_{l}^{n}\right)\right],
\end{array}
$$

where $\bar{U}_{k}=\left(u_{1 k}, \ldots, u_{m k}\right), \bar{U}_{l}^{n}=\left(\bar{u}_{1 l}^{n}, \ldots, \bar{u}_{m l}^{n}\right)$, and $w_{l k}$ is defined by (3.22).

Step 3. If $\max _{1 \leq i \leq m}\left|\bar{u}_{i l}^{n+1}-\bar{u}_{i l}^{n}\right| \leq \varepsilon$, then let $\bar{u}_{i l}^{n+1}=\tilde{u}_{i l}$ and stop the iteration, otherwise set $n:=n+1$, go to Step 2 . 


\section{Convergence and Error Estimation}

In this section we will analyze the convergence of Algorithm 3.2 proposed in Section 3. The proof of convergence rate of Algorithm 3.3 is similar. When $t=t_{l}$, the system can be expressed as

$$
\begin{aligned}
u_{i}\left(t_{l}\right)= & y_{i}\left(t_{l}\right)+\sum_{j=1}^{m}\left[h \sum_{k=0}^{l-1} w_{l k}\left(t_{l}-t_{k}\right)^{\alpha_{i j}}\left(\ln \left|t_{l}-t_{k}\right|\right)^{\beta_{i j}} k_{i j}\left(t_{l}, t_{k}, U_{k}\right)+w_{l l} h^{1+\alpha_{i j}} k_{i j}\left(t_{l}, t_{l}, U_{l}\right)\right] \\
& +\sum_{j=1}^{m}\left[E_{i j, l, t}\left(\left(t_{l}-t\right)^{\alpha_{i j}}\left(\ln \left|t_{l}-t\right|\right)^{\beta_{i j}} k_{i j}\left(t_{l}, t, u(t)\right)\right)\right] .
\end{aligned}
$$

By Lemma 3.1, the remainder has the following estimate:

$$
\sum_{j=1}^{m}\left[E_{i j, l, t}\left(\left(t_{l}-t\right)^{\alpha_{i j}}\left(\ln \left|t_{l}-t\right|\right)^{\beta_{i j}} k_{i j}\left(t_{l}, t, u(t)\right)\right)\right]=O\left(h^{2+\alpha}(\ln h)^{\beta}\right),
$$

where $\alpha=\min _{1 \leq i, j \leq m} \alpha_{i j}>-1$ and $\beta=\max _{1 \leq i, j \leq m} \beta_{i j}$.

Letting $e_{i l}=u_{i}\left(t_{l}\right)-u_{i l}$ and $\left\{u_{i l}\right\}$ be the solution of the discrete equations, we derive

$$
\begin{aligned}
e_{i 0}= & 0, \\
e_{i l}= & \sum_{j=1}^{m} \sum_{k=0}^{l-1} h w_{l k}\left(t_{l}-t_{k}\right)^{\alpha_{i j}}\left(\ln \left|t_{l}-t_{k}\right|\right)^{\beta_{i j}} \\
& \times\left(k_{i j}\left(t_{l}, t_{k}, u_{1}\left(t_{k}\right), \ldots, u_{m}\left(t_{k}\right)\right)-k_{i j}\left(t_{l}, t_{k}, u_{1 k}, \ldots, u_{m k}\right)\right) \\
& +\sum_{j=1}^{m} w_{l l} h^{1+\alpha_{i j}}\left(k_{i j}\left(t_{l}, t_{l}, u_{1}\left(t_{l}\right), \ldots, u_{m}\left(t_{l}\right)\right)-k_{i j}\left(t_{l}, t_{l}, u_{1 l}, \ldots, u_{m l}\right)\right) \\
& +\sum_{j=1}^{m}\left[E_{i j, l, t}\left(\left(t_{l}-t\right)^{\alpha_{i j}}\left(\ln \left|t_{l}-t\right|\right)^{\beta_{i j}} k_{i j}\left(t_{l}, t, u_{1}(t), \ldots, u_{m}(t)\right)\right)\right], \quad i=1,2, \ldots, m, l=1,2, \ldots, N .
\end{aligned}
$$

Lemma 4.1. Suppose that the sequence $\left\{e_{i}\right\}_{i=1}^{N}$ satisfies

$$
\begin{gathered}
e_{0}=0, \\
\left|e_{i}\right| \leq \sum_{j=1}^{i-1} B_{i j}\left|e_{j}\right|+A, \quad 1 \leq i \leq N,
\end{gathered}
$$

where $B_{i j}=2 \operatorname{Lh}\left(t_{i}-t_{j}\right)^{\alpha}\left(\ln \left|t_{i}-t_{j}\right|\right)^{\beta},-1<\alpha \leq 0, \beta=0,1$, and $h$ is sufficiently small such that $L h w_{i i} \leq 1 / 2$. Then

$$
\left|e_{i}\right| \leq H A,
$$

where $H=\sum_{k=0}^{\infty} R^{k} /(k !)^{s}, R=2 L(b-a)^{s} \Gamma(s) e^{1 / 12 s}(e / s) h^{s}$, and $s=1+\alpha$ 
Theorem 4.2. Assume that $h$ is sufficiently small, then the system of the nonlinear discrete equation (3.19) has a unique solution and the simple iteration (3.24) is geometrically convergent.

Proof. Firstly, if $U_{l}=\left(u_{1 l}, u_{2 l}, \ldots, u_{m l}\right)$ and $V_{l}=\left(v_{1 l}, v_{2 l}, \ldots, v_{m l}\right)(l=1,2, \ldots, N)$ are solutions to (3.19), then $\left\{Z_{l}=U_{l}-V_{l}\right\}$ satisfies the inequality

$$
\begin{aligned}
&\left|u_{i l}-v_{i l}\right| \leq \sum_{j=1}^{m}\left[h \sum_{k=0}^{l-1} w_{l k}\left(t_{l}-t_{k}\right)^{\alpha_{i j}}\left(\ln \left|t_{l}-t_{k}\right|\right)^{\beta_{i j}}\left|k_{i j}\left(t_{l}, t_{k}, U_{k}\right)-k_{i j}\left(t_{l}, t_{k}, V_{k}\right)\right|\right] \\
&+\sum_{j=1}^{m}\left[w_{l l} h^{1+\alpha_{i j}}\left|k_{i j}\left(t_{l}, t_{l}, U_{l}\right)-k_{i j}\left(t_{l}, t_{k}, V_{l}\right)\right|\right] \\
& \leq \sum_{j=1}^{m}\left[h \sum_{k=0}^{l-1} w_{l k}\left(t_{l}-t_{k}\right)^{\alpha_{i j}}\left(\ln \left|t_{l}-t_{k}\right|\right)^{\beta_{i j}} L\left\|U_{k}-V_{k}\right\|_{\infty}+w_{l l} h^{1+\alpha_{i j}} L\left\|U_{l}-V_{l}\right\|_{\infty}\right], \\
& i=1,2, \ldots, m, \quad l=1,2, \ldots, N,
\end{aligned}
$$

where we use $u_{i 0}=v_{i 0}=y\left(t_{i}\right)$. Note that $\left\|Z_{k}\right\|_{\infty}=\max _{1 \leq j \leq m}\left|z_{j k}\right|$, then we easily deduce that

$$
\begin{gathered}
\left\|Z_{0}\right\|_{\infty}=0, \\
\left\|Z_{l}\right\|_{\infty} \leq M_{1} h \sum_{k=0}^{l-1}\left(t_{l}-t_{k}\right)^{\alpha}\left(\ln \left|t_{l}-t_{k}\right|\right)^{\beta}\left\|Z_{k}\right\|_{\infty}, \quad l=1,2, \ldots, N,
\end{gathered}
$$

where $M_{1}=m \max _{0 \leq k<l \leq N}\left|w_{l k}\left(t_{l}-t_{k}\right)^{\alpha_{i j}-\alpha}(1+c)\left(\ln \left|t_{l}-t_{k}\right|\right)^{\beta} L_{i j}\right|$.

If $h$ is sufficiently small such that $M_{1} h^{1+\alpha} \leq 1 / 2$, we have

$$
\begin{gathered}
\left\|Z_{0}\right\|_{\infty}=0 \\
\left\|Z_{l}\right\|_{\infty} \leq 2 M_{1} h \sum_{k=0}^{l-1}\left(t_{l}-t_{k}\right)^{\alpha}\left(\ln \left|t_{l}-t_{k}\right|\right)^{\beta}\left\|Z_{k}\right\|_{\infty}, \quad l=1,2, \ldots, N .
\end{gathered}
$$

Then by Lemma 4.1 , we get $\left\|Z_{l}\right\|_{\infty}=0, l=1,2, \ldots, N$. Hence the uniqueness is shown.

Secondly, from the iteration (3.24) we have

$$
\begin{aligned}
\left|u_{i l}^{n+1}-u_{i l}^{n}\right| & =\sum_{j=1}^{m}\left[w_{l l} h^{1+\alpha_{i j}} \mid k_{i j}\left(t_{l}, t_{l}, U_{l}^{n}\right)-k_{i j}\left(t_{l}, t_{l}, U_{l}^{n-1}\right) \|\right] \\
& \leq \sum_{j=1}^{m}\left[w_{l l} h^{1+\alpha_{i j}} L\left\|U_{l}^{n}-U_{l}^{n-1}\right\|_{\infty}\right], \quad i=1,2, \ldots, m ; l=1,2, \ldots, N .
\end{aligned}
$$

Then

$$
\left\|U_{l}^{n+1}-U_{l}^{n}\right\|_{\infty} \leq M h^{1+\alpha}\left\|U_{l}^{n}-U_{l}^{n-1}\right\|_{\infty} \leq \frac{1}{2}\left\|U_{l}^{n}-U_{l}^{n-1}\right\|_{\infty}, \quad l=1,2, \ldots, N,
$$


where $M=m w_{l l} L$. We assume that $h$ is small enough such that $M h^{1+\alpha} \leq 1 / 2$. Thus we prove that the simple iteration (3.24) is geometrically convergent, and its limit is the unique solution of (3.19).

Theorem 4.3. There is a positive constant $C$ independent of $h$ such that

$$
\left\|U_{l}-\tilde{U}_{l}\right\|_{\infty} \leq C \varepsilon h^{1+\alpha}
$$

where $\tilde{U}_{l}=\left(\tilde{u}_{1 l}, \tilde{u}_{2 l}, \ldots, \tilde{u}_{m l}\right)=\left(u_{1 l}^{n+1}, u_{2 l}^{n+1}, \ldots, u_{m l}^{n+1}\right)$ is defined in Algorithm 3.2.

Proof. Letting $V_{l}=U_{l}-\tilde{U}_{l}$, we get

$$
\begin{aligned}
u_{i 0}-\tilde{u}_{i 0}= & 0, \\
u_{i l}-\tilde{u}_{i l}= & \sum_{j=1}^{m}\left[h \sum_{k=0}^{l-1} w_{l k}\left(t_{l}-t_{k}\right)^{\alpha_{i j}}\left(\ln \left|t_{l}-t_{k}\right|\right)^{\beta_{i j}}\left(k_{i j}\left(t_{l}, t_{k}, U_{k}\right)-k_{i j}\left(t_{l}, t_{k}, \tilde{U}_{k}\right)\right)\right] \\
& +\sum_{j=1}^{m} w_{l l} h^{1+\alpha_{i j}}\left(k_{i j}\left(t_{l}, t_{l}, U_{l}\right)-k_{i j}\left(t_{l}, t_{l}, U_{l}^{n}\right)\right) \\
= & \sum_{j=1}^{m}\left[h \sum_{k=0}^{l-1} w_{l k}\left(t_{l}-t_{k}\right)^{\alpha_{i j}}\left(\ln \left|t_{l}-t_{k}\right|\right)^{\beta_{i j}}\left(k_{i j}\left(t_{l}, t_{k}, U_{k}\right)-k_{i j}\left(t_{l}, t_{k}, \tilde{U}_{k}\right)\right)\right] \\
& +\sum_{j=1}^{m} w_{l l} h^{1+\alpha_{i j}}\left(k_{i j}\left(t_{l}, t_{l}, U_{l}\right)-k_{i j}\left(t_{l}, t_{l}, U_{l}^{n+1}\right)\right) \\
& +\sum_{j=1}^{m} w_{l l} h^{1+\alpha_{i j}}\left(k_{i j}\left(t_{l}, t_{l}, U_{l}^{n+1}\right)-k_{i j}\left(t_{l}, t_{l}, U_{l}^{n}\right)\right), \quad i=1,2, \ldots, m ; l=1,2, \ldots, N .
\end{aligned}
$$

By Algorithm 3.2 and Lipschitz condition, we have

$$
\left\|V_{l}\right\|_{\infty} \leq M_{1} h \sum_{k=0}^{l-1}\left(t_{l}-t_{k}\right)^{\alpha}\left(\ln \left|t_{l}-t_{k}\right|\right)^{\beta}\left\|V_{k}\right\|_{\infty}+2 M_{1} \varepsilon h^{1+\alpha}
$$

Using Lemma 3.1, we get $\left\|U_{l}-\tilde{U}_{l}\right\|_{\infty} \leq C \varepsilon h^{1+\alpha}$.

Theorem 4.4. There is a positive constant $C$ independent of $h$ such that the errors $e_{i l}=u_{i}\left(t_{l}\right)-u_{i l}(i=$ $1,2, \ldots, m, l=1,2, \ldots, N)$ have the following error bound:

$$
\max _{0 \leq l \leq N ; 1 \leq i \leq m}\left|e_{i l}\right| \leq C h^{2+\alpha}|\ln h|^{\beta}
$$


Proof. Taking $E_{l}=\max _{1 \leq i \leq m}\left|e_{i l}\right|$, we get

$$
\begin{gathered}
E_{0}=0, \\
E_{l} \leq M_{1} h \sum_{k=0}^{l-1}\left(t_{l}-t_{k}\right)^{\alpha}\left(\ln \left|t_{l}-t_{k}\right|\right)^{\beta} E_{k}+M_{1} h^{1+\alpha} E_{l}+O\left(h^{2+\alpha}\right), \quad l=1,2, \ldots, N .
\end{gathered}
$$

If $h$ is sufficiently small such that $M_{1} h^{1+\alpha} \leq 1 / 2$, we have

$$
E_{l} \leq 2 M_{1} h \sum_{k=0}^{l-1}\left(t_{l}-t_{k}\right)^{\alpha}\left(\ln \left|t_{l}-t_{k}\right|\right)^{\beta} E_{k}+O\left(h^{2+\alpha}\right), \quad l=1,2, \ldots, N
$$

Using inequality (4.5), we can get

$$
\max _{0 \leq l \leq N ; 1 \leq i \leq m}\left|e_{i l}\right| \leq C h^{2+\alpha}|\ln h|^{\beta} .
$$

Corollary 4.5. Assume that $\varepsilon=O(h)$ in Algorithm 3.2, one can obtain the estimate

$$
\max _{1 \leq i \leq m ; 1 \leq l \leq N}\left|u_{i}\left(t_{l}\right)-\tilde{u}_{i l}\right|=O\left(h^{2+\alpha}(\ln h)^{\beta}\right) .
$$

\section{Asymptotic Expansion, Combination, and a Posteriori Error Estimate}

In the following we only derive the asymptotic expansions of the errors and the a posteriori error estimation of Algorithm 3.2. For Algorithm 3.3 we just simply present the corresponding result.

From Lemma 3.1 and (4.1) by using Taylor's expansion, we have

$$
\begin{aligned}
u_{i}\left(t_{l}\right)= & y_{i}\left(t_{l}\right)+\sum_{j=1}^{m}\left[h \sum_{k=0}^{l-1} w_{l k}\left(t_{l}-t_{k}\right)^{\alpha_{i j}}\left(\ln \left|t_{l}-t_{k}\right|\right)^{\beta_{i j}} k_{i j}\left(t_{l}, t_{k}, U_{k}\right)+w_{l l} h^{1+\alpha_{i j}} k_{i j}\left(t_{l}, t_{l}, U_{l}\right)\right] \\
& +h^{2+\alpha}(\ln h)^{\beta} \sum_{j=1}^{m} T_{i j}^{0}\left(t_{l}\right)+h^{2+\alpha} \sum_{j=1}^{m} T_{i j}^{1}\left(t_{l}\right)+O\left(h^{2+\alpha_{1}}|\ln h|^{\beta_{1}}\right), \quad i=1, \ldots, m,
\end{aligned}
$$

where $\alpha_{1}=\min _{1 \leq i, j \leq m}\left\{\alpha_{i j}: \alpha_{i j}>\alpha\right\}, \beta_{1}=\max _{1 \leq i, j \leq m}\left\{\beta_{i j}: \alpha_{i j}=\alpha_{1}\right\}$, and

$$
\begin{aligned}
& T_{i j}^{0}(t)= \begin{cases}-\zeta(-\alpha-1) \frac{d}{d t} k_{i j}\left(t_{l}, t, u(t)\right) & \text { if } \alpha_{i j}=\alpha, \\
0 & \text { if } \alpha_{i j}>\alpha,\end{cases} \\
& T_{i j}^{1}(t)= \begin{cases}\beta \zeta^{\prime}(-\alpha-1) \frac{d}{d t} k_{i j}\left(t_{l}, t, u(t)\right) & \text { if } \alpha_{i j}=\alpha, \\
0 & \text { if } \alpha_{i j}>\alpha .\end{cases}
\end{aligned}
$$


For $e_{i l}=u_{i}\left(t_{l}\right)-u_{i l}$, we have

$$
\begin{aligned}
e_{i 0}= & u_{i}\left(t_{0}\right)-u_{i 0}=0, \\
e_{i l}= & u_{i}\left(t_{l}\right)-u_{i l} \\
= & \sum_{j=1}^{m} \sum_{k=0}^{l-1} h w_{l k}\left(t_{l}-t_{k}\right)^{\alpha_{i j}}\left(\ln \left|t_{l}-t_{k}\right|\right)^{\beta_{i j}}\left(k_{i j}\left(t_{l}, t_{k}, u\left(t_{k}\right)\right)-k_{i j}\left(t_{l}, t_{k}, U_{k}\right)\right) \\
& +\sum_{j=1}^{m} w_{l l} h^{1+\alpha_{i j}}\left(k_{i j}\left(t_{l}, t_{l}, u\left(t_{l}\right)\right)-k_{i j}\left(t_{l}, t_{l}, U_{l}\right)\right)+h^{2+\alpha}(\ln h)^{\beta} T_{i}^{0}\left(t_{l}\right) \\
& +h^{2+\alpha} T_{i}^{1}\left(t_{l}\right)+O\left(h^{2+\alpha_{1}}|\ln h|^{\beta_{1}}\right), \quad i=1,2, \ldots, m, l=1,2, \ldots, N .
\end{aligned}
$$

Here $\sum_{j=1}^{m} T_{i j}^{k}=T_{i}^{k}\left(t_{l}\right), k=0,1$.

Using Theorem 4.4 and Taylor's expansion, we get

$$
\begin{aligned}
k_{i j}\left(t_{l}, t_{k}, u_{1}\left(t_{k}\right), \ldots, u_{m}\left(t_{k}\right)\right)-k_{i j}\left(t_{l}, t_{k}, u_{1 k}, \ldots, u_{m k}\right) \\
=k_{i j, u_{1}}\left(t_{l}, t_{k}, u_{1}\left(t_{k}\right), \ldots, u_{m}\left(t_{k}\right)\right)\left(u_{1}\left(t_{k}\right)-u_{1 k}\right)+\cdots \\
\quad+k_{i j, u_{m}}\left(t_{l}, t_{k}, u_{1}\left(t_{k}\right), \ldots, u_{m}\left(t_{k}\right)\right)\left(u_{m}\left(t_{k}\right)-u_{m k}\right)+o\left(h^{2}\right) \\
=k_{i j, u_{1}}\left(t_{l}, t_{k}, u_{1}\left(t_{k}\right), \ldots, u_{m}\left(t_{k}\right)\right) e_{1 k}+\ldots \\
\quad+k_{i j, u_{m}}\left(t_{l}, t_{k}, u_{1}\left(t_{k}\right), \ldots, u_{m}\left(t_{k}\right)\right) e_{m k}+o\left(h^{2}\right),
\end{aligned}
$$

where we assume that $k_{i j}(s, t, u)$ is derivable for $u_{l}, l=1, \ldots, m$ and let

$$
k_{i j, u_{l}}(s, t, u)=\frac{\partial}{\partial u_{l}} k_{i j}(s, t, u), \quad l=1, \ldots, m .
$$

Then

$$
\begin{aligned}
e_{i 0}= & u_{i}\left(t_{0}\right)-u_{i 0}=0, \\
e_{i l}= & u_{i}\left(t_{l}\right)-u_{i l} \\
= & \sum_{j=1}^{m}\left[h \sum_{k=0}^{l-1} w_{l k}\left(t_{l}-t_{k}\right)^{\alpha_{i j}}\left(\ln \left|t_{l}-t_{k}\right|\right)^{\beta_{i j}} \sum_{r=1}^{m} k_{i j, u_{r}}\left(t_{l}, t_{k}, u\left(t_{k}\right)\right) e_{r k}\right] \\
& +\sum_{j=1}^{m} w_{l l} h^{1+\alpha_{i j}} \sum_{r=1}^{m} k_{i j, u_{r}}\left(t_{l}, t_{l}, u\left(t_{l}\right)\right) e_{r l}+h^{2+\alpha}(\ln h)^{\beta} T_{i}^{0}\left(t_{l}\right)+h^{2+\alpha} T_{i}^{1}\left(t_{l}\right) \\
& +O\left(h^{2+\alpha_{1}}|\ln h|^{\beta_{1}}\right), \quad i=1,2, \ldots, m, l=1,2, \ldots, N .
\end{aligned}
$$

Obviously if $\beta=0$, then $T_{i}^{1}\left(t_{l}\right)=0$. 
Now we construct the following auxiliary system of linear Volterra integral equations: find $\left\{\Phi_{j}^{r}(s), j=1,2, \ldots, m, r=0,1\right\}$ satisfying

$\Phi_{i}^{r}(t)=T_{i}^{r}(t)+\sum_{j=1}^{m} \int_{0}^{t}(t-s)^{\alpha_{i j}}(\ln |t-s|)^{\beta_{i j}}\left[\sum_{p=1}^{m} k_{i j, u_{p}}(t, s, u(s)) \Phi_{1}^{r}(s)\right], \quad i=1,2, \ldots, m, r=0,1$,

and their discrete system of equations: find $\left\{\Phi_{i l}^{r}, i=1,2, \ldots, m, l=1, \ldots, N\right\}$ satisfying

$$
\begin{aligned}
\Phi_{i 0}^{r}= & 0, \\
\Phi_{i l}^{r}= & T_{i}^{r}\left(t_{l}\right)+\sum_{j=1}^{m}\left[h \sum_{k=0}^{l-1} w_{l k}\left(t_{l}-t_{k}\right)^{\alpha_{i j}}\left(\ln \left|t_{l}-t_{k}\right|\right)^{\beta_{i j}} \sum_{p=1}^{m} k_{i j, u_{p}}\left(t_{l}, t_{k}, u\left(t_{k}\right)\right) \Phi_{p k}^{r}\right] \\
& +\sum_{j=1}^{m} w_{l l} h^{1+\alpha_{i j}} \sum_{p=1}^{m} k_{i j, u_{p}}\left(t_{l}, t_{l}, u\left(t_{k}\right)\right) \Phi_{p k^{\prime}}^{r} \quad i=1,2, \ldots, m, l=1,2, \ldots, N ; r=0,1 .
\end{aligned}
$$

From Theorem 4.4, we have

$$
\max _{1 \leq i \leq m, 1 \leq l \leq N}\left|\Phi_{i l}^{r}-\Phi_{i}^{r}\left(t_{l}\right)\right|=O\left(h^{2+\alpha_{1}}|\ln h|^{\beta_{1}}\right), \quad r=0,1 .
$$

Substituting (5.8) and (5.7) into (5.6), we get

$$
\begin{aligned}
E_{i 0}= & 0, \\
E_{i l}= & \sum_{j=1}^{m}\left[h \sum_{k=0}^{l-1} w_{l k}\left(t_{l}-t_{k}\right)^{\alpha_{i j}} k_{i j, u_{l}}\left(t_{l}, t_{k}, u_{j}\left(t_{k}\right)\right) E_{j k}\right]+\sum_{j=1}^{m} w_{l l} h^{1+\alpha_{i j}} k_{i j, u_{l}}\left(t_{l}, t_{l}, u_{j}\left(t_{l}\right)\right) E_{j l} \\
& +O\left(h^{2+\alpha_{1}}(\ln h)^{\beta_{1}}\right), \quad i=1,2, \ldots, m, l=1,2, \ldots, N .
\end{aligned}
$$

Note that $E_{i l}=e_{i l}-h^{2+\alpha}(\ln h)^{\beta} T_{i l}^{0}-h^{2+\alpha} T_{i l}^{1}$. Using Lemma 4.1, we get

$u_{i}\left(t_{l}\right)-u_{i l}-h^{2+\alpha}(\ln h)^{\beta} \Phi_{i l}^{0}\left(t_{l}\right)-h^{2+\alpha} \Phi_{i l}^{1}\left(t_{l}\right)=O\left(h^{2+\alpha_{1}}(\ln h)^{\beta_{1}}\right), \quad i=1,2, \ldots, m, l=1,2, \ldots, N$

From (5.9) we obtain

$$
u_{i}\left(t_{l}\right)-u_{i l}-h^{2+\alpha}(\ln h)^{\beta} \Phi_{i}^{0}\left(t_{l}\right)-h^{2+\alpha} \Phi_{i}^{1}\left(t_{l}\right)=O\left(h^{2+\alpha_{1}}|\ln h|^{\beta_{1}}\right), \quad i=1,2, \ldots, m, l=1,2, \ldots, N,
$$


where $\Phi_{i}^{1}(t)=0$ if $\beta=0$. Similarly for Algorithm 3.3 we have

$$
u_{i}\left(t_{l}\right)-\bar{u}_{i l}-h^{2+\alpha}(\ln h)^{\beta} \bar{\Phi}_{i l}^{0}-h^{2+\alpha} \bar{\Phi}_{i l}^{1}=O\left(h^{2+\alpha_{1}}|\ln h|^{\beta_{1}}\right)
$$

or

$$
u_{i}\left(t_{l}\right)-\bar{u}_{i l}-h^{2+\alpha}(\ln h)^{\beta} \bar{\Phi}_{i}^{0}\left(t_{l}\right)-h^{2+\alpha} \bar{\Phi}_{i}^{1}\left(t_{l}\right)=O\left(h^{2+\alpha_{1}}|\ln h|^{\beta_{1}}\right), \quad i=1,2, \ldots, m, l=1,2, \ldots, N,
$$

where $\bar{\Phi}_{i}^{1}(t)=0$ if $\beta=0$ and

$$
\begin{gathered}
\bar{\Phi}_{i}^{1}\left(t_{l}\right)=-\left[2^{-\alpha-1} \ln 2 \frac{\zeta(-\alpha-1)}{\zeta^{\prime}(-\alpha-1)}+\left(2^{-\alpha-1}-1\right)\right] \Phi_{i}^{1}\left(t_{l}\right), \\
\bar{\Phi}_{i}^{0}\left(t_{l}\right)=\left(2^{-\alpha-1}-1\right) \Phi_{i}^{0}\left(t_{l}\right) .
\end{gathered}
$$

From the above discussion, we have proved the following theorem for the combination method.

Theorem 5.1. (1) If $\beta=0$, then

$$
u_{i l}^{c}=\frac{\left(1-2^{-1-\alpha}\right) u_{i l}+\bar{u}_{i l}}{2-2^{-1-\alpha}}=u_{i}\left(t_{l}\right)+O\left(h^{2+\alpha_{1}}\right), \quad i=1,2, \ldots, m, l=1,2, \ldots, N .
$$

Furthermore, one has the following a posteriori error estimate:

$$
\min \left\{u_{i l}, \bar{u}_{i l}\right\} \leq u_{i l}^{c} \leq \max \left\{u_{i l}, \bar{u}_{i l}\right\} \quad \text { or } \quad\left|u_{i}\left(t_{l}\right)-\frac{u_{i l}+\bar{u}_{i l}}{2}\right| \leq\left|\frac{u_{i l}-\bar{u}_{i l}}{2}\right|
$$

That is, one can estimate the average errors $\left|u_{i}\left(t_{l}\right)-\left(u_{i l}+\bar{u}_{i l}\right) / 2\right|$ by $\left|\left(u_{i l}-\bar{u}_{i l}\right) / 2\right|$.

(2) If $\beta=1$, then

$$
u_{i l}^{c}=\frac{\left(1-2^{-1-\alpha}\right) u_{i l}+\bar{u}_{i l}}{2-2^{-1-\alpha}}=u_{i}\left(t_{l}\right)+O\left(h^{2+\alpha}\right), \quad i=1,2, \ldots, m, l=1,2, \ldots, N .
$$

Hence one can obtain a high order of accuracy, which is better than $O\left(h^{2+\alpha}(\ln h)\right)$. One also easily deduces that the modified mid-point rectangular quadrature method is better than modified trapezoidal quadrature method when $1-2^{-1-\alpha} \rightarrow 0$.

\section{Numerical Examples}

In this section, we present two numerical examples to illustrate the features of the combination method discussed in this paper. Let $E_{T}$ denote the error of modified trapezoidal quadrature method, $E_{M}$ the error of modified mid-point rectangular quadrature method, and $E_{C}$ the error of combination method. 
Table 1: The errors and the a posterior error estimate of $x(s)$ in Example 6.1 at mesh point $(h=1 / 10$, $\alpha=-1 / 2$ in combination coefficient).

\begin{tabular}{cccccc}
\hline $\mathrm{s}$ & $E_{T}$ & $E_{M}$ & $E_{C}$ & $\left|x\left(s_{i}\right)-\left(T x_{i}+M x_{i}\right) / 2\right|$ & $\left|\left(T x_{i}-M x_{i}\right) / 2\right|$ \\
\hline 0.1 & $-3.871 E-03$ & $9.519 E-04$ & $-1.406 E-04$ & $1.459 E-03$ & $2.411 E-03$ \\
0.2 & $-4.559 E-03$ & $1.313 E-03$ & $-1.764 E-05$ & $1.623 E-03$ & $2.936 E-03$ \\
0.3 & $-4.664 E-03$ & $1.381 E-03$ & $1.129 E-05$ & $1.642 E-03$ & $3.022 E-03$ \\
0.4 & $-4.758 E-03$ & $1.428 E-03$ & $2.692 E-05$ & $1.665 E-03$ & $3.093 E-03$ \\
0.5 & $-4.908 E-03$ & $1.480 E-03$ & $3.323 E-05$ & $1.714 E-03$ & $3.194 E-03$ \\
0.6 & $-5.130 E-03$ & $1.546 E-03$ & $3.369 E-05$ & $1.792 E-03$ & $3.338 E-03$ \\
0.7 & $-5.434 E-03$ & $1.630 E-03$ & $2.996 E-05$ & $1.902 E-03$ & $3.532 E-03$ \\
0.8 & $-5.824 E-03$ & $1.735 E-03$ & $2.281 E-05$ & $2.044 E-03$ & $3.779 E-03$ \\
0.9 & $-6.310 E-03$ & $1.864 E-03$ & $1.249 E-05$ & $2.223 E-03$ & $4.087 E-03$ \\
1.0 & $-6.904 E-03$ & $2.021 E-03$ & $-1.165 E-06$ & $2.442 E-03$ & $4.462 E-03$ \\
\hline Maximum error & $6.904 E-03$ & $2.021 E-03$ & $1.406 E-04$ & $2.442 E-03$ & $4.462 E-03$ \\
\hline
\end{tabular}

Table 2: The errors and the a posterior error estimate of $x(s)$ in Example 6.1 at mesh point $(h=1 / 20$, $\alpha=-1 / 2$ in combination coefficient).

\begin{tabular}{cccccc}
\hline $\mathrm{s}$ & $E_{T}$ & $E_{M}$ & $E_{C}$ & $\left|x\left(s_{i}\right)-\left(T x_{i}+M x_{i}\right) / 2\right|$ & $\left|\left(T x_{i}-M x_{i}\right) / 2\right|$ \\
\hline 0.1 & $-1.526 E-03$ & $4.376 E-04$ & $-7.358 E-06$ & $5.444 E-04$ & $9.820 E-04$ \\
0.2 & $-1.560 E-03$ & $4.709 E-04$ & $1.082 E-05$ & $5.446 E-04$ & $1.016 E-03$ \\
0.3 & $-1.557 E-03$ & $4.786 E-04$ & $1.750 E-05$ & $5.391 E-04$ & $1.018 E-03$ \\
0.4 & $-1.576 E-03$ & $4.871 E-04$ & $1.963 E-05$ & $5.446 E-04$ & $1.032 E-03$ \\
0.5 & $-1.625 E-03$ & $5.013 E-04$ & $1.957 E-05$ & $5.620 E-04$ & $1.063 E-03$ \\
0.6 & $-1.705 E-03$ & $5.228 E-04$ & $1.821 E-05$ & $5.909 E-04$ & $1.114 E-03$ \\
0.7 & $-1.815 E-03$ & $5.522 E-04$ & $1.596 E-05$ & $6.314 E-04$ & $1.184 E-03$ \\
0.8 & $-1.958 E-03$ & $5.901 E-04$ & $1.297 E-05$ & $6.837 E-04$ & $1.274 E-03$ \\
0.9 & $-2.135 E-03$ & $6.373 E-04$ & $9.312 E-06$ & $7.487 E-04$ & $1.386 E-03$ \\
1.0 & $-2.350 E-03$ & $6.948 E-04$ & $4.944 E-06$ & $8.278 E-04$ & $1.523 E-03$ \\
\hline Maximum error & $2.350 E-03$ & $6.948 E-04$ & $1.963 E-05$ & $8.278 E-04$ & $1.523 E-03$ \\
\hline Maximum error ratios & 2.937 & 2.908 & 7.162 & 2.950 & 2.931 \\
\hline
\end{tabular}

Example 6.1. Consider the following system of integral equations with algebraic singularity:

$$
\begin{gathered}
x(s)=\frac{9}{5} s^{5 / 3}+s-\int_{0}^{s} \frac{1}{\sqrt[3]{s-t}}(x(t)+y(t)) d t, \quad 0 \leq s \leq 1, \\
y(s)=\frac{\pi}{2} s-\frac{16}{15} s^{5 / 2}+s+\int_{0}^{s} \frac{1}{\sqrt{s-t}}\left(\frac{1}{2} x^{2}(t)+\frac{1}{2} y^{2}(t)-\sqrt{y(t)}\right) d t, \quad 0 \leq s \leq 1
\end{gathered}
$$

with the exact solution $x(s)=s, y(s)=s$.

The errors of the approximation solutions obtained by Algorithms 3.2 and 3.3 and their combination are presented in Tables 1, 2, 3, and 4. Numerical results show that the combination method has higher order convergence rate than the two original algorithms. Tables 1-4 also show that the error ratios of Algorithms 3.2 and 3.3 are close to the theoretic value, which is $2^{2+(-1 / 2)}=2.828$ and the error ratio of the combination method is better than the $2^{2+(-1 / 2)}$. 
Table 3: The errors and the a posterior error estimate of $y(s)$ in Example 6.1 at mesh point $(h=1 / 10$, $\alpha=-1 / 2$ in combination coefficient).

\begin{tabular}{cccccc}
\hline $\mathrm{s}$ & $E_{T}$ & $E_{M}$ & $E_{C}$ & $\left|y\left(s_{i}\right)-\left(T y_{i}+M y_{i}\right) / 2\right|$ & $\left|\left(T y_{i}-M y_{i}\right) / 2\right|$ \\
\hline 0.1 & $7.256 E-03$ & $-2.096 E-03$ & $2.280 E-05$ & $2.580 E-03$ & $4.676 E-03$ \\
0.2 & $4.454 E-03$ & $-1.246 E-03$ & $4.548 E-05$ & $1.604 E-03$ & $2.850 E-03$ \\
0.3 & $4.207 E-03$ & $-1.184 E-03$ & $3.705 E-05$ & $1.511 E-03$ & $2.696 E-03$ \\
0.4 & $4.472 E-03$ & $-1.254 E-03$ & $4.324 E-05$ & $1.609 E-03$ & $2.863 E-03$ \\
0.5 & $4.976 E-03$ & $-1.388 E-03$ & $5.327 E-05$ & $1.794 E-03$ & $3.182 E-03$ \\
0.6 & $5.646 E-03$ & $-1.568 E-03$ & $6.621 E-05$ & $2.039 E-03$ & $3.607 E-03$ \\
0.7 & $6.465 E-03$ & $-1.787 E-03$ & $8.219 E-05$ & $2.339 E-03$ & $4.126 E-03$ \\
0.8 & $7.436 E-03$ & $-2.046 E-03$ & $1.017 E-04$ & $2.695 E-03$ & $4.741 E-03$ \\
0.9 & $8.577 E-03$ & $-2.350 E-03$ & $1.257 E-04$ & $3.114 E-03$ & $5.463 E-03$ \\
1.0 & $9.917 E-03$ & $-2.704 E-03$ & $1.555 E-04$ & $3.607 E-03$ & $6.310 E-03$ \\
\hline Maximum error & $9.917 E-03$ & $2.704 E-03$ & $1.555 E-04$ & $3.607 E-03$ & $6.310 E-03$ \\
\hline
\end{tabular}

Table 4: The errors and the a posterior error estimate of $y(s)$ in Example 6.1 at mesh point $(h=1 / 20$, $\alpha=-1 / 2$ in combination coefficient).

\begin{tabular}{|c|c|c|c|c|c|}
\hline $\mathrm{s}$ & $E_{T}$ & $E_{M}$ & $E_{C}$ & $\left|y\left(s_{i}\right)-\left(T y_{i}+M y_{i}\right) / 2\right|$ & $\left|\left(T y_{i}-M y_{i}\right) / 2\right|$ \\
\hline 0.1 & $1.748 E-03$ & $-4.874 E-04$ & $1.914 E-05$ & $6.305 E-04$ & $1.118 E-03$ \\
\hline 0.2 & $1.298 E-03$ & $-3.659 E-04$ & $1.110 E-05$ & $4.662 E-04$ & $8.321 E-04$ \\
\hline 0.3 & $1.311 E-03$ & $-3.680 E-04$ & $1.234 E-05$ & $4.715 E-04$ & $8.396 E-04$ \\
\hline 0.4 & $1.450 E-03$ & $-4.058 E-04$ & $1.467 E-05$ & $5.222 E-04$ & $9.280 E-04$ \\
\hline 0.5 & $1.657 E-03$ & $-4.622 E-04$ & $1.778 E-05$ & $5.972 E-04$ & $1.059 E-03$ \\
\hline 0.6 & $1.915 E-03$ & $-5.328 E-04$ & $2.164 E-05$ & $6.909 E-04$ & $1.224 E-03$ \\
\hline 0.7 & $2.221 E-03$ & $-6.166 E-04$ & $2.628 E-05$ & $8.023 E-04$ & $1.419 E-03$ \\
\hline 0.8 & $2.579 E-03$ & $-7.143 E-04$ & $3.179 E-05$ & $9.324 E-04$ & $1.647 E-03$ \\
\hline 0.9 & $2.995 E-03$ & $-8.277 E-04$ & $3.834 E-05$ & $1.084 E-03$ & $1.912 E-03$ \\
\hline 1.0 & $3.481 E-03$ & $-9.597 E-04$ & $4.618 E-05$ & $1.260 E-03$ & $2.220 E-03$ \\
\hline Maximum error & $3.481 E-03$ & $9.597 E-04$ & $4.618 E-05$ & $1.260 E-03$ & $2.220 E-03$ \\
\hline Maximum error ratios & 2.817 & 2.800 & 3.137 & 2.824 & 2.814 \\
\hline
\end{tabular}

Example 6.2. Consider the following system of integro-differential equations with algebraic singularity:

$$
\begin{gathered}
x^{\prime}(s)=\frac{5}{28} x(s)^{7 / 5}-\frac{125}{84} s^{12 / 5}+1+\int_{0}^{s} \frac{1}{\sqrt[5]{(s-t)^{3}}}\left(x^{2}(t)-\frac{1}{10} x(t)\right) d t, \quad 0 \leq s \leq 1, \\
x(0)=0
\end{gathered}
$$

with the exact solution $x(s)=s$. Let

$$
\begin{gathered}
u(s)=x(s) \\
v(s)=\frac{5}{28} x^{7 / 5}(s)-\frac{125}{84} s^{12 / 5}+1+\int_{0}^{s} \frac{1}{\sqrt[5]{(s-t)^{3}}}\left(x^{2}(t)-\frac{1}{10} x(t)\right) d t .
\end{gathered}
$$


Table 5: The errors and the a posterior error estimate of $x(s)$, that is, $u(s)$ in Example 6.2 at mesh point ( $h=1 / 10, \alpha=-3 / 5$ in combination coefficient).

\begin{tabular}{cccccc}
\hline $\mathrm{s}$ & $E_{T}$ & $E_{M}$ & $E_{C}$ & $\left|u\left(s_{i}\right)-\left(T u_{i}+M u_{i}\right) / 2\right|$ & $\left|\left(T u_{i}-M u_{i}\right) / 2\right|$ \\
\hline 0.1 & $5.977 E-05$ & $-1.630 E-05$ & $-1.470 E-06$ & $2.174 E-05$ & $3.804 E-05$ \\
0.2 & $2.767 E-04$ & $-7.095 E-05$ & $-3.177 E-06$ & $1.029 E-04$ & $1.738 E-04$ \\
0.3 & $7.027 E-04$ & $-1.738 E-04$ & $-2.926 E-06$ & $2.645 E-04$ & $4.382 E-04$ \\
0.4 & $1.374 E-03$ & $-3.342 E-04$ & $-1.214 E-06$ & $5.199 E-04$ & $8.541 E-04$ \\
0.5 & $2.348 E-03$ & $-5.659 E-04$ & $2.229 E-06$ & $8.912 E-04$ & $1.457 E-03$ \\
0.6 & $3.717 E-03$ & $-8.898 E-04$ & $8.219 E-06$ & $1.414 E-03$ & $2.303 E-03$ \\
0.7 & $5.621 E-03$ & $-1.338 E-03$ & $1.830 E-05$ & $2.141 E-03$ & $3.480 E-03$ \\
0.8 & $8.278 E-03$ & $-1.961 E-03$ & $3.525 E-05$ & $3.159 E-03$ & $5.119 E-03$ \\
0.9 & $1.202 E-02$ & $-2.832 E-03$ & $6.397 E-05$ & $4.595 E-03$ & $7.427 E-03$ \\
1.0 & $1.737 E-02$ & $-4.066 E-03$ & $1.134 E-04$ & $6.653 E-03$ & $1.072 E-02$ \\
\hline Maximum error & $1.737 E-02$ & $4.066 E-03$ & $1.134 E-04$ & $6.653 E-03$ & $1.072 E-02$ \\
\hline
\end{tabular}

Table 6: The errors and the a posterior error estimate of $x(s)$, that is, $u(s)$ in Example 6.2 at mesh point ( $h=1 / 20, \alpha=-3 / 5$ in combination coefficient).

\begin{tabular}{|c|c|c|c|c|c|}
\hline $\mathrm{s}$ & $E_{T}$ & $E_{M}$ & $E_{C}$ & $\left|u\left(s_{i}\right)-\left(T u_{i}+M u_{i}\right) / 2\right|$ & $\left|\left(T u_{i}-M u_{i}\right) / 2\right|$ \\
\hline 0.1 & $1.576 E-05$ & $-5.207 E-06$ & $-1.119 E-06$ & $5.279 E-06$ & $1.049 E-05$ \\
\hline 0.2 & $9.596 E-05$ & $-2.543 E-05$ & $-1.763 E-06$ & $3.527 E-05$ & $6.069 E-05$ \\
\hline 0.3 & $2.555 E-04$ & $-6.436 E-05$ & $-2.019 E-06$ & $9.554 E-05$ & $1.599 E-04$ \\
\hline 0.4 & $5.067 E-04$ & $-1.252 E-04$ & $-2.052 E-06$ & $1.907 E-04$ & $3.159 E-04$ \\
\hline 0.5 & $8.701 E-04$ & $-2.130 E-04$ & $-1.870 E-06$ & $3.286 E-04$ & $5.416 E-04$ \\
\hline 0.6 & $1.379 E-03$ & $-3.356 E-04$ & $-1.398 E-06$ & $5.216 E-04$ & $8.571 E-04$ \\
\hline 0.7 & $2.083 E-03$ & $-5.049 E-04$ & $-4.622 E-07$ & $7.890 E-04$ & $1.294 E-03$ \\
\hline 0.8 & $3.060 E-03$ & $-7.394 E-04$ & $1.267 E-06$ & $1.160 E-03$ & $1.900 E-03$ \\
\hline 0.9 & $4.428 E-03$ & $-1.067 E-03$ & $4.395 E-06$ & $1.680 E-03$ & $2.747 E-03$ \\
\hline 1.0 & $6.366 E-03$ & $-1.529 E-03$ & $1.003 E-05$ & $2.419 E-03$ & $3.948 E-03$ \\
\hline Maximum error & $6.366 E-03$ & $1.529 E-03$ & $1.003 E-05$ & $2.419 E-03$ & $3.948 E-03$ \\
\hline Maximum error ratio & 2.729 & 2.659 & 11.307 & 2.751 & 2.715 \\
\hline
\end{tabular}

Then the original system of integro-differential equations can be transformed to the following system of Volterra integral equations:

$$
\begin{gathered}
u(s)=\int_{0}^{s} v(t) d t, \quad u(0)=0, \\
v(s)=\frac{5}{28} u^{7 / 5}(s)-\frac{125}{84} s^{12 / 5}+1+\int_{0}^{s} \frac{1}{\sqrt[5]{(s-t)^{3}}}\left(u^{2}(t)-\frac{1}{10} u(t)\right) d t, \quad v(0)=1 .
\end{gathered}
$$

The two equations contain algebraic singularity with the coefficient $\alpha=-3 / 5$. The exact solution is

$$
\begin{aligned}
& u(s)=s, \\
& v(s)=1 .
\end{aligned}
$$


The errors of the numerical solutions obtained by Algorithms 3.2 and 3.3 and their combination are presented in Tables 5 and 6. Numerical results still show that the combination method has obviously higher convergence rate than the two algorithms, as well as better maximum error ratio, which is greater than $2^{2+(-3 / 5)}=2.639$.

\section{Conclusions}

In this paper we use the combination method to solve the systems of integral and integrodifferential equations with weakly singular kernels of the second kind, which are important to many applications but have few results. High accuracy and high parallelism are two features of this method. Our numerical results also confirm the theoretical conclusions.

\section{Acknowledgment}

This work was supported by the National Natural Science Foundation of China (10671136).

\section{References}

[1] H. Brunner, "The numerical solution of weakly singular Volterra integral equations by collocation on graded meshes," Mathematics of Computation, vol. 45, no. 172, pp. 417-437, 1985.

[2] H. Brunner, "Polynomial spline collocation methods for Volterra integrodifferential equations with weakly singular kernels," IMA Journal of Numerical Analysis, vol. 6, no. 2, pp. 221-239, 1986.

[3] K. E. Atkinson, The Numerical Solution of Integral Equations of the Second Kind, vol. 4 of Cambridge Monographs on Applied and Computational Mathematics, Cambridge University Press, Cambridge, UK, 1997.

[4] W. Hackbusch, Integralgleichungen, Teubner Studienbücher Mathematik, B. G. Teubner, Stuttgart, Germany, 1989.

[5] Z. Chen, Y. Xu, and J. Zhao, "The discrete Petrov-Galerkin method for weakly singular integral equations," Journal of Integral Equations and Applications, vol. 11, no. 1, pp. 1-35, 1999.

[6] T. Lü and H. Yong, "Extrapolation method for solving weakly singular nonlinear Volterra integral equations of the second kind," Journal of Mathematical Analysis and Applications, vol. 324, no. 1, pp. 225-237, 2006.

[7] A. Pedas and E. Tamme, "Spline collocation method for integro-differential equations with weakly singular kernels," Journal of Computational and Applied Mathematics, vol. 197, no. 1, pp. 253-269, 2006.

[8] A. Pedas and E. Tamme, "Discrete Galerkin method for Fredholm integro-differential equations with weakly singular kernels," Journal of Computational and Applied Mathematics, vol. 213, no. 1, pp. 111-126, 2008.

[9] H. Brunner, Y. Lin, and S. Zhang, "Higher accuracy methods for second-kind Volterra integral equations based on asymptotic expansions of iterated Galerkin methods," Journal of Integral Equations and Applications, vol. 10, no. 4, pp. 375-396, 1998.

[10] L. Qun and T. Lü, "The combination of approximate solutions for accelerating the convergence," RAIRO Analyse Numérique, vol. 18, no. 2, pp. 153-160, 1984.

[11] Y. Liu and T. Lü, "High accuracy combination algorithm and a posteriori error estimation for solving the first kind Abel integral equations," Applied Mathematics and Computation, vol. 178, no. 2, pp. 441451, 2006.

[12] J. N. Lyness and B. W. Ninham, "Numerical quadrature and asymptotic expansions," Mathematics of Computation, vol. 21, pp. 162-178, 1967.

[13] T. Lü and Y. Huang, "A generalization of discrete Gronwall inequality and its application to weakly singular Volterra integral equation of the second kind," Journal of Mathematical Analysis and Applications, vol. 282, no. 1, pp. 56-62, 2003.

[14] Y. Liu and T. Lü, "Mechanical quadrature methods and their extrapolation for solving first kind Abel integral equations," Journal of Computational and Applied Mathematics, vol. 201, no. 1, pp. 300-313, 2007. 
[15] J. Navot, "A further extension of Euler-Maclaurin summation formula," Journal of Mathematical Physics, vol. 41, pp. 155-184, 1962.

[16] T. Lü and Y. Huang, "A high accuracy combinatorial algorithm and a posteriori error estimation for solving second-kind Abel integral equations," Journal of Systems Science and Mathematical Sciences, vol. 24, no. 1, pp. 110-117, 2004. 


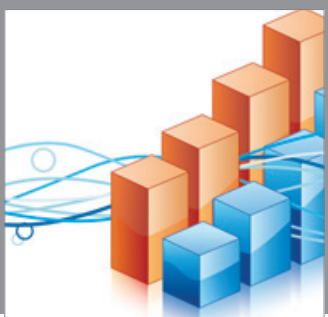

Advances in

Operations Research

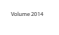

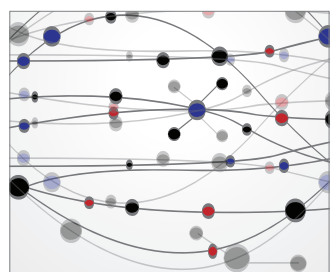

\section{The Scientific} World Journal
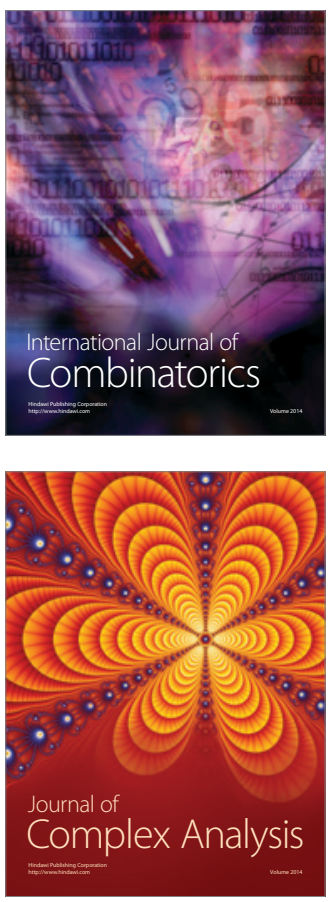

International Journal of

Mathematics and

Mathematical

Sciences
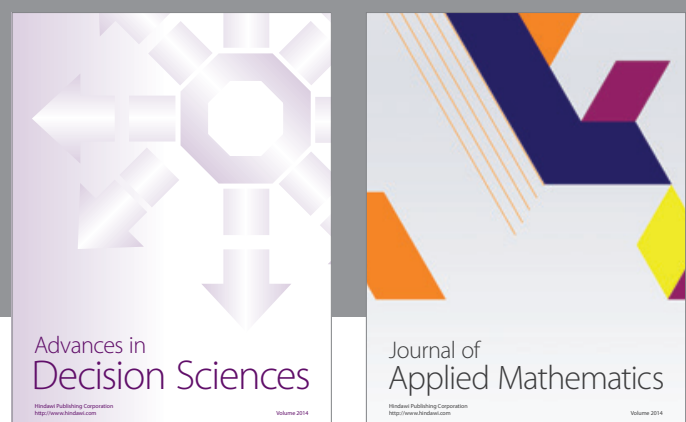

Journal of

Applied Mathematics
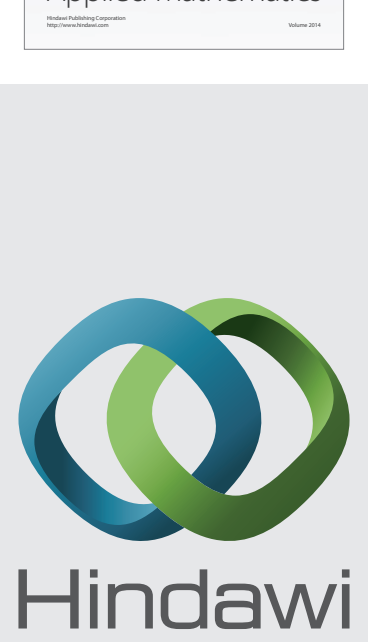

Submit your manuscripts at http://www.hindawi.com
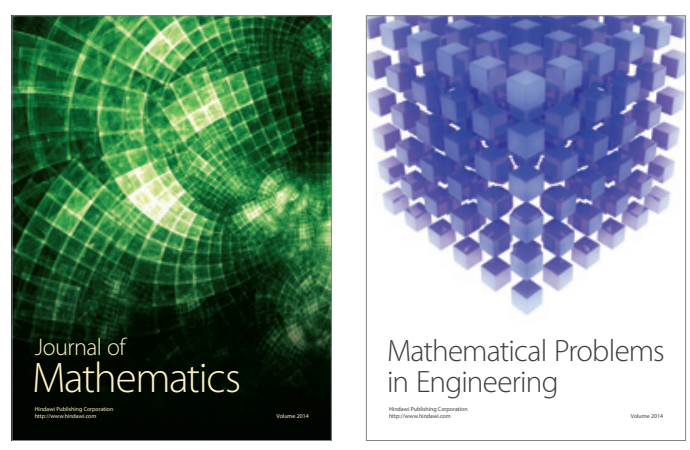

Mathematical Problems in Engineering
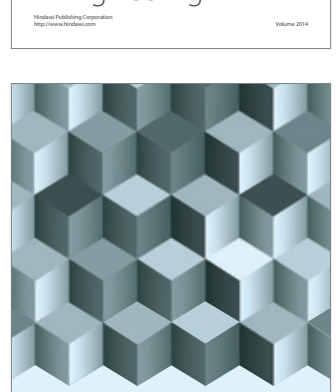

Journal of

Function Spaces
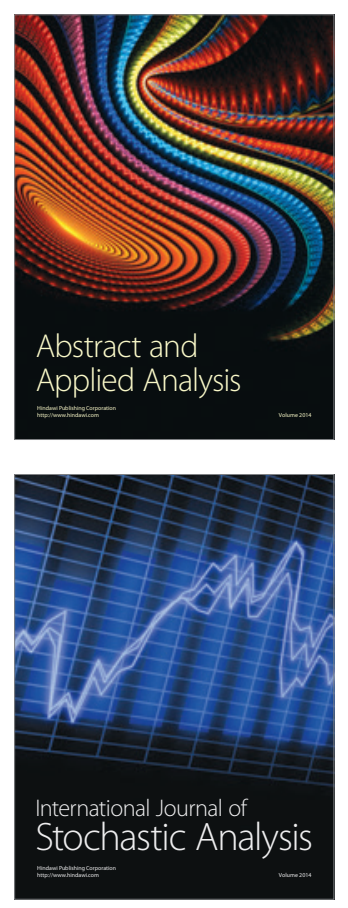

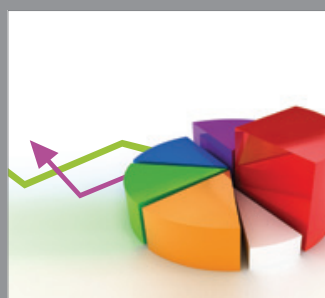

ournal of

Probability and Statistics

Promensencen
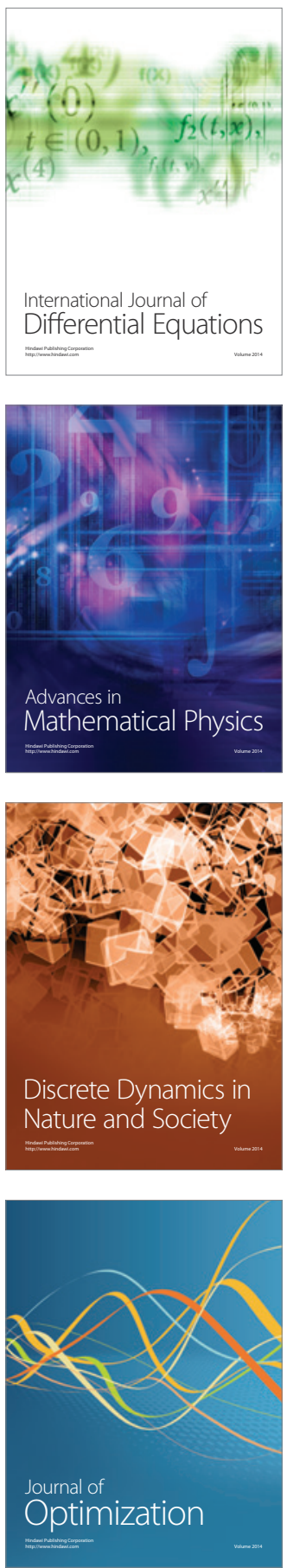